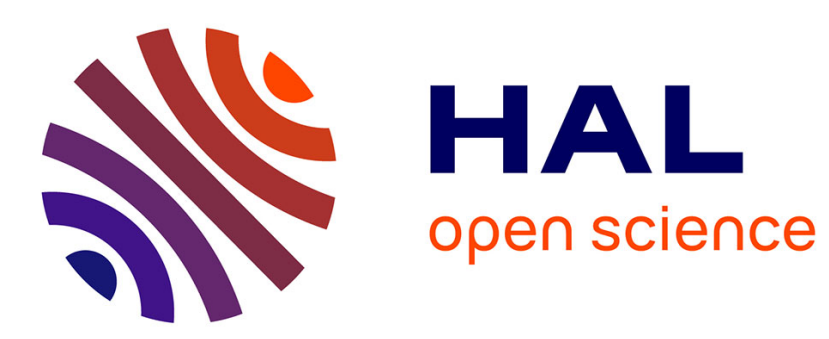

\title{
Sliding mode multiple observer for fault detection and isolation
}

\author{
Abdelkader Akhenak, Mohammed Chadli, Didier Maquin, José Ragot
}

\section{To cite this version:}

Abdelkader Akhenak, Mohammed Chadli, Didier Maquin, José Ragot. Sliding mode multiple observer for fault detection and isolation. 42th IEEE Conference on Decision and Control, Dec 2003, Hawaii, United States. pp.953-958, 10.1109/CDC.2003.1272690 • hal-00151307

\section{HAL Id: hal-00151307 https://hal.science/hal-00151307}

Submitted on 25 Mar 2014

HAL is a multi-disciplinary open access archive for the deposit and dissemination of scientific research documents, whether they are published or not. The documents may come from teaching and research institutions in France or abroad, or from public or private research centers.
L'archive ouverte pluridisciplinaire HAL, est destinée au dépôt et à la diffusion de documents scientifiques de niveau recherche, publiés ou non, émanant des établissements d'enseignement et de recherche français ou étrangers, des laboratoires publics ou privés. 


\title{
Sliding mode multiple observer for fault detection and isolation
}

\author{
Abdelkader Akhenak, Mohammed Chadli, Didier Maquin, José Ragot \\ Centre de Recherche en Automatique de Nancy - CNRS UMR 7039 \\ Institut National Polytechnique de Lorraine \\ 2, avenue de la forêt de Haye - 54516 Vandoeuvre-lès-Nancy cedex \\ \{aakhenak, mchadli, dmaquin, jragot\}@ensem.inpl-nancy.fr
}

\begin{abstract}
This paper deals with the design of a sliding mode multiple observer (an observer based on a multiple model) allowing to estimate the state vector of a non linear dynamical system. This latter is influenced by unknown inputs which act on it through a known transmission matrix. The state estimation and consequently the output estimation can therefore be classically used for detecting and isolating faults.
\end{abstract}

Keywords: multiple model, multiple observer, sliding mode, state estimation, unknown inputs.

\section{Introduction}

The general procedure for using an observer for fault detection and isolation consists of three main steps:

1. Estimating the output measurement of the system by using an adapted structure of the observer.

2. Comparing the estimated and the measured outputs, i.e. generating the so-called residuals.

3. Analyzing the residuals and deciding if a fault occurred or not.

The decision process may be based on a simple threshold test applied on the instantaneous value or on a moving average of the residuals. However, when the system under consideration is subject to unknown disturbances or unknown inputs, to properly achieve fault detection needs the effect of the disturbance to be de-coupled from the residual signal; that allows to avoid false alarms in the detection procedure. This problem is known in the literature as the robust fault detection problem which is mainly solved by using unknown input observers [7].

The problem of state estimation of linear systems subject to unknown inputs has received considerable attention [4] and [11]. However, a very few works have been developed for nonlinear systems [3] and [12]. The purpose of this work is to propose a methodology for the design of a nonlinear observer of this type of systems.

\section{Sliding mode multiple observer}

The concept of sliding mode emerged from the Soviet Union in the late sixties where the effects of introducing discontinuous control action into dynamical systems were explored. By the use of a judicious switched control law, it was found that the system states could be forced to reach and subsequently remain on a pre-defined surface in the state space. Whilst constrained to this surface, the resulting reduced-order motion - referred to as the sliding motion - was shown to be insensitive to any uncertainty or external disturbance signals which were implicit in the input of the system.

This inherent robustness property has resulted in world wide interest and research in the area of sliding mode control. These ideas have subsequently been employed in other situations including the problem of state estimation via an observer.

The earliest work of Utkin is based on a discontinuous structure for the observer as described in [5]. Walcott and Żak use a Lyapunov-based approach to formulate and synthesize an observer which, under appropriate assumptions, exhibits asymptotic state error decay in the presence of bounded nonlinearities and uncertainties on the input of the system [4]. Edwards and Spurgeon propose an observer strategy, similar in style to that of Walcott and Żak, which circumvents the use of a symbolic manipulation and offers an explicit design algorithm. Within the framework of the multiple model approach, the synthesis of regulators by using sliding mode was also considered [10].

The presented work consists in conceiving a sliding mode multiple observer, capable of reconstructing the state and the output vectors of a system when some inputs are unknown, such as each local observer is modeled in the same way of Walcott's and Żak's observer (1988).

\subsection{Multiple model representation}

Let us consider a nonlinear system represented by the following multiple model (with $\mathrm{r}$ local models) with unknown inputs:

$\left\{\begin{array}{l}\dot{\mathrm{x}}(\mathrm{t})=\sum_{\mathrm{i}=1}^{\mathrm{r}} \mu_{\mathrm{i}}(\xi(\mathrm{t}))\left(\mathrm{A}_{\mathrm{i}} \mathrm{x}(\mathrm{t})+\mathrm{B}_{\mathrm{i}} \mathrm{u}(\mathrm{t})+\mathrm{R}_{\mathrm{i}} \overline{\mathrm{u}}(\mathrm{t})+\mathrm{D}_{\mathrm{i}}\right) \\ \mathrm{y}(\mathrm{t})=\mathrm{Cx}(\mathrm{t})\end{array}\right.$

where $\mathrm{x}(\mathrm{t}) \in \mathrm{R}^{\mathrm{n}}$ is the state vector, $\mathrm{u}(\mathrm{t}) \in \mathrm{R}^{\mathrm{m}}$ is the input vector, $\overline{\mathrm{u}}(\mathrm{t}) \in \mathrm{R}^{\mathrm{q}}$ the vector of unknown inputs and $y(t) \in R^{p}$ the vector of measurable output. For the ith local model, $A_{i} \in R^{n \times n}$ is the state matrix, $B_{i} \in R^{n \times m}$ is the matrix of input, $R_{i} \in R^{n \times q}$ is the distribution matrix of the unknown inputs and $D_{i} \in R^{n \times l}$ is a matrix 
depending on the operating point. Moreover, $\mathrm{C} \in \mathrm{R}^{\mathrm{p} \times \mathrm{n}}$ is the matrix of output. It is assumed that the matrices $R_{i}$ are perfectly known; on the contrary the time evolution of $\bar{u}(t)$ is unknown. Finally, $\xi(t)$ represents the vector of decision depending on the input and/or the measurable state variables: the value of $\xi(t)$ allows to specify what are the active local models at time $t$.

The procedure that allows to obtain this structure and to estimate its parameters is not developed here. Let us only state that one can either uses techniques of parametric estimation [8] or linearization techniques [9].

\subsection{Multiple observer structure}

The proposed observer for the multiple model (1), is a linear combination of local observers, each of them having the structure proposed by Walcott and Żak.

In this part, we consider that the inputs $\overline{\mathrm{u}}(\mathrm{t})$ are bounded, such as $\|\overline{\mathrm{u}}(\mathrm{t})\| \leq \rho$, where $\rho$ is scalar and $\|\cdot\|$ represents the Euclidean norm.

It is also assumed that there exists matrices $G_{i} \in R^{n \times p}$ such that $A_{0 i}=A_{i}-G_{i} C$ have stable eigenvalues and that there exists Lyapunov pairs $\left(P, Q_{i}\right)$ such that the structural constraints:

$\mathrm{A}_{0 \mathrm{i}}^{\mathrm{T}} \mathrm{P}+\mathrm{PA}_{0 \mathrm{i}}=-\mathrm{Q}_{\mathrm{i}}$

$\mathrm{C}^{\mathrm{T}} \mathrm{F}_{\mathrm{i}}^{\mathrm{T}}=\mathrm{PR}_{\mathrm{i}}, \quad \forall \mathrm{i} \in\{1, \ldots, \mathrm{r}\}$

are satisfied for some $F_{i} \in R^{q \times p}$.

The proposed observer has the form:

$\left\{\begin{array}{l}\dot{\hat{x}}(t)=\sum_{i=1}^{\mathrm{r}} \mu_{\mathrm{i}}(\xi(\mathrm{t}))\left(\begin{array}{l}\mathrm{A}_{\mathrm{i}} \hat{\mathrm{x}}(\mathrm{t})+\mathrm{B}_{\mathrm{i}} \mathrm{u}(\mathrm{t})- \\ \mathrm{G}_{\mathrm{i}}(\mathrm{C} \hat{\mathrm{x}}(\mathrm{t})-\mathrm{y}(\mathrm{t}))+\mathrm{K}_{\mathrm{i}} \mathrm{v}_{\mathrm{i}}(\mathrm{t})\end{array}\right) \\ \hat{\mathrm{y}}(\mathrm{t})=\mathrm{C} \hat{\mathrm{x}}(\mathrm{t})\end{array}\right.$

One can determine the matrices $G_{i}$ and the control variables $v_{i}(t)$, with $v_{i}(t) \in R^{q}$, which guarantee the exponential convergence of $\hat{x}(t)$ towards $x(t)$.

Let us note that equation (2) allows to isolate the unknown inputs.

In order to estimate the state vector of the system (1), we are going to proceed to two successive coordinate changes of the state vector.

\subsection{First change of coordinates}

Let us suppose that all the pairs $\left(\mathrm{A}_{\mathrm{i}}, \mathrm{C}\right)$ are observable. As the outputs of the system are to be considered for the design of the observer, it is logical to effect a coordinates change so that the outputs directly appear as components of the new state vector. Without loss of generality, the output distribution matrix can always be written as:

$\mathrm{C}=\left[\begin{array}{ll}\mathrm{C}_{1} & \mathrm{C}_{2}\end{array}\right]$

where $\quad \mathrm{C}_{1} \in \mathrm{R}^{\mathrm{p} \times(\mathrm{n}-\mathrm{p})}, \mathrm{C}_{2} \in \mathrm{R}^{\mathrm{p} \times \mathrm{p}}$ and $\operatorname{det}\left(\mathrm{C}_{2}\right) \neq 0 . \quad$ The following change of coordinates is then operated: $\tilde{\mathrm{x}}(\mathrm{t})=\tilde{\mathrm{T}} \mathrm{x}(\mathrm{t})$,

$\tilde{\mathrm{T}}=\left[\begin{array}{cc}\mathrm{I}_{\mathrm{n}-\mathrm{p}} & 0 \\ \mathrm{C}_{1} & \mathrm{C}_{2}\end{array}\right]$ where $\tilde{\mathrm{T}}$ is a non singular matrix. With respect to this new coordinate system, the new output distribution matrix can be written as:

$\widetilde{\mathrm{C}}=\mathrm{C} \tilde{\mathrm{T}}^{-1}$

The other system matrices are written as:

$\tilde{\mathrm{A}}_{\mathrm{i}}=\tilde{\mathrm{T}} \mathrm{A}_{\mathrm{i}} \tilde{\mathrm{T}}^{-1}=\left[\begin{array}{cc}\tilde{\mathrm{A}}_{11 \mathrm{i}} & \tilde{\mathrm{A}}_{12 \mathrm{i}} \\ \tilde{\mathrm{A}}_{21 \mathrm{i}} & \tilde{\mathrm{A}}_{22 \mathrm{i}}\end{array}\right], \tilde{\mathrm{B}}_{\mathrm{i}}=\tilde{\mathrm{T}}_{\mathrm{i}}=\left[\begin{array}{c}\tilde{\mathrm{B}}_{1 \mathrm{i}} \\ \tilde{\mathrm{B}}_{2 \mathrm{i}}\end{array}\right]$

$\tilde{\mathrm{D}}_{\mathrm{i}}=\tilde{\mathrm{T}} \mathrm{D}_{\mathrm{i}}=\left[\begin{array}{c}\tilde{\mathrm{D}}_{1 \mathrm{i}} \\ \tilde{\mathrm{D}}_{2 \mathrm{i}}\end{array}\right], \tilde{\mathrm{R}}_{\mathrm{i}}=\tilde{\mathrm{T}}_{\mathrm{i}}=\left[\begin{array}{c}\tilde{\mathrm{R}}_{1 \mathrm{i}} \\ \tilde{\mathrm{R}}_{2 \mathrm{i}}\end{array}\right]$

The Lyapunov matrices $\left(\mathrm{P}, \mathrm{Q}_{\mathrm{i}}\right)$ and the structural constraints (2) became, in the new coordinates, as follows:

$\tilde{\mathrm{P}}=\left(\tilde{\mathrm{T}}^{-1}\right)^{\mathrm{T}} \mathrm{P} \tilde{\mathrm{T}}^{-1}$

$\tilde{\mathrm{Q}}_{\mathrm{i}}=\left(\tilde{\mathrm{T}}^{-1}\right)^{\mathrm{T}} \mathrm{Q}_{\mathrm{i}} \tilde{\mathrm{T}}^{-1}$

$\tilde{\mathrm{C}}^{\mathrm{T}} \mathrm{F}_{\mathrm{i}}^{\mathrm{T}}=\tilde{\mathrm{P}} \tilde{\mathrm{R}}_{\mathrm{i}}$

According to definitions (7), the system (1) can be rewritten under the following form:

$\left\{\begin{array}{l}\dot{\tilde{x}}(t)=\sum_{i=1}^{r} \mu_{i}(\xi(t))\left(\tilde{\mathrm{A}}_{\mathrm{i}} \tilde{\mathrm{x}}(\mathrm{t})+\tilde{\mathrm{B}}_{\mathrm{i}} \mathrm{u}(\mathrm{t})+\tilde{\mathrm{R}}_{\mathrm{i}} \overline{\mathrm{u}}(\mathrm{t})+\tilde{\mathrm{D}}_{\mathrm{i}}\right) \\ \mathrm{y}(\mathrm{t})=\tilde{\mathrm{x}}_{2}(\mathrm{t})\end{array}\right.$

where $\tilde{\mathrm{x}}(\mathrm{t})=\tilde{\mathrm{T}} \mathrm{x}(\mathrm{t})$

Summarizing, the change of coordinates allows to express directly the output vector as a part of the state vector.

\subsection{Isolating the unknown inputs}

Now the result concerning the conception of a robust observer in the presence of unknown inputs established by Walcott and Żak may be used. This result is then extended to the conception of a multiple observer.

Let the local models $\left(\tilde{\mathrm{A}}_{\mathrm{i}}, \tilde{\mathrm{B}}_{\mathrm{i}}, \tilde{\mathrm{R}}_{\mathrm{i}}, \tilde{\mathrm{C}}\right)$ defined by equation (9) where $\tilde{\mathrm{A}}_{\mathrm{i}}$ are stable matrices $\forall \mathrm{i} \in\{1, \ldots, \mathrm{r}\}$, and $\left(\overline{\mathrm{A}}_{\mathrm{i}}, \overline{\mathrm{B}}_{\mathrm{i}}, \overline{\mathrm{R}}_{\mathrm{i}}, \overline{\mathrm{C}}\right)$ be related to $\left(\tilde{\mathrm{A}}_{\mathrm{i}}, \tilde{\mathrm{B}}_{\mathrm{i}}, \tilde{\mathrm{R}}_{\mathrm{i}}, \tilde{\mathrm{C}}\right)$ by a nonsingular similarity transformation $\overline{\mathrm{T}}$, where $\overline{\mathrm{x}}(\mathrm{t})=\overline{\mathrm{T}} \tilde{\mathrm{x}}(\mathrm{t})$. Then, the system matrices are written in the new base as follows [4]:

$\left\{\begin{array}{l}\overline{\mathrm{A}}_{\mathrm{i}}=\overline{\mathrm{T}} \tilde{\mathrm{A}}_{\mathrm{i}} \overline{\mathrm{T}}^{-1}=\left[\begin{array}{ll}\overline{\mathrm{A}}_{11 \mathrm{i}} & \overline{\mathrm{A}}_{12 \mathrm{i}} \\ \overline{\mathrm{A}}_{21 \mathrm{i}} & \overline{\mathrm{A}}_{22 \mathrm{i}}\end{array}\right], \overline{\mathrm{B}}_{\mathrm{i}}=\overline{\mathrm{T}} \tilde{\mathrm{B}}_{\mathrm{i}}=\left[\begin{array}{c}\overline{\mathrm{B}}_{1 \mathrm{i}} \\ \overline{\mathrm{B}}_{2 \mathrm{i}}\end{array}\right] \\ \overline{\mathrm{D}}_{\mathrm{i}}=\overline{\mathrm{T}} \tilde{\mathrm{D}}_{\mathrm{i}}=\left[\begin{array}{l}\overline{\mathrm{D}}_{1 \mathrm{i}} \\ \overline{\mathrm{D}}_{2 \mathrm{i}}\end{array}\right], \overline{\mathrm{R}}_{\mathrm{i}}=\overline{\mathrm{T}} \tilde{\mathrm{R}}_{\mathrm{i}}=\left[\begin{array}{l}\overline{\mathrm{R}}_{1 \mathrm{i}} \\ \overline{\mathrm{R}}_{2 \mathrm{i}}\end{array}\right] \text { et } \overline{\mathrm{C}}=\tilde{\mathrm{C}}^{-1}\end{array}\right.$

Proposition 1: let $\left(\tilde{\mathrm{A}}_{\mathrm{i}}, \tilde{\mathrm{B}}_{\mathrm{i}}, \tilde{\mathrm{R}}_{\mathrm{i}}, \tilde{\mathrm{C}}\right)$ be a local model for which there exists a pair $\left(\tilde{\mathrm{P}}, \mathrm{F}_{\mathrm{i}}\right)$ defined by constraints (8c). Then, there exists a non-singular similarity transformation $\overline{\mathrm{T}}$ so that the quadruple $\left(\overline{\mathrm{A}}_{\mathrm{i}}, \overline{\mathrm{B}}_{\mathrm{i}}, \overline{\mathrm{R}}_{\mathrm{i}}, \overline{\mathrm{C}}\right)$ in the new coordinates exhibits the following properties: 
1. $\overline{\mathrm{A}}_{0 \mathrm{i}}=\overline{\mathrm{A}}_{\mathrm{i}}-\overline{\mathrm{G}}_{\mathrm{i}} \overline{\mathrm{C}}=\left[\begin{array}{ll}\overline{\mathrm{A}}_{011 \mathrm{i}} & \overline{\mathrm{A}}_{012 \mathrm{i}} \\ \overline{\mathrm{A}}_{021 \mathrm{i}} & \overline{\mathrm{A}}_{022 \mathrm{i}}\end{array}\right]$ where

$\overline{\mathrm{A}}_{011 \mathrm{i}} \in \mathrm{R}^{(\mathrm{n}-\mathrm{p}) \times(\mathrm{n}-\mathrm{p})}$ are stable that implies that

$\overline{\mathrm{A}}_{11 \mathrm{i}} \in \mathrm{R}^{(\mathrm{n}-\mathrm{p}) \times(\mathrm{n}-\mathrm{p})}$ are stable.

2. $\overline{\mathrm{R}}_{\mathrm{i}}=\left[\begin{array}{c}0 \\ \mathrm{P}_{22}^{*} \mathrm{~F}_{\mathrm{i}}^{\mathrm{T}}\end{array}\right]$ where $\mathrm{P}_{22}^{*} \in \mathrm{R}^{\mathrm{p} \times \mathrm{p}}$ with $\mathrm{P}_{22}^{*}=\left(\mathrm{P}_{22}^{*}\right)^{\mathrm{T}}>0$

3. $\overline{\mathrm{C}}=\tilde{\mathrm{C}} \overline{\mathrm{T}}^{-1}=\left[\begin{array}{ll}0 & \mathrm{I}_{\mathrm{p}}\end{array}\right]$

4. $\overline{\mathrm{P}}=\left(\overline{\mathrm{T}}^{-1}\right)^{\mathrm{T}} \tilde{\mathrm{P}} \overline{\mathrm{T}}^{-1}=\left[\begin{array}{cc}\overline{\mathrm{P}}_{1} & 0 \\ 0 & \overline{\mathrm{P}}_{2}\end{array}\right]$ where $\overline{\mathrm{P}}_{1} \in \mathrm{R}^{(\mathrm{n}-\mathrm{p}) \times(\mathrm{n}-\mathrm{p})}$ and $\overline{\mathrm{P}}_{2} \in \mathrm{R}^{\mathrm{p} \times \mathrm{p}}$.

Proof: Let us consider the pairs $\left(\tilde{\mathrm{P}}, \mathrm{F}_{\mathrm{i}}\right)$ associated to the local model $\left(\tilde{\mathrm{A}}_{\mathrm{i}}, \widetilde{\mathrm{B}}_{\mathrm{i}}, \tilde{\mathrm{R}}_{\mathrm{i}}, \widetilde{\mathrm{C}}\right)$ and the Lyapunov matrix $\tilde{\mathrm{P}}$ written as follows:

$\tilde{\mathrm{P}}=\left[\begin{array}{ll}\tilde{\mathrm{P}}_{11} & \tilde{\mathrm{P}}_{12} \\ \tilde{\mathrm{P}}_{12}^{T} & \tilde{\mathrm{P}}_{22}\end{array}\right]$ where $\left\{\begin{array}{l}\tilde{\mathrm{P}}_{11} \in \mathrm{R}^{(\mathrm{n}-\mathrm{p}) \times(\mathrm{n}-\mathrm{p})} \text { is regular } \\ \tilde{\mathrm{P}}_{12} \in \mathrm{R}^{(\mathrm{n}-\mathrm{p}) \times \mathrm{p}} \text { and } \tilde{\mathrm{P}}_{22} \in \mathrm{R}^{\mathrm{p} \times \mathrm{p}}\end{array}\right.$

Let us define the change of coordinates using the following transformation:

$\overline{\mathrm{T}}=\left[\begin{array}{cc}\tilde{\mathrm{P}}_{11} & \tilde{\mathrm{P}}_{12} \\ 0 & \mathrm{I}_{\mathrm{p}}\end{array}\right]$

which is non-singular since $\operatorname{det}(\overline{\mathrm{T}})=\operatorname{det}\left(\widetilde{\mathrm{P}}_{11}\right) \neq 0$ because $\tilde{\mathrm{P}}_{11}=\tilde{\mathrm{P}}_{11}^{\mathrm{T}}>0$. In the new coordinate system, $\overline{\mathrm{C}}=\tilde{\mathrm{C}} \overline{\mathrm{T}}^{-1}=\left[\begin{array}{ll}0 & \mathrm{I}_{\mathrm{p}}\end{array}\right]$ and thus property 3 is satisfied.

From equation (8), one obtains $\tilde{\mathrm{R}}_{\mathrm{i}}=\tilde{\mathrm{P}}^{-1} \tilde{\mathrm{C}}^{\mathrm{T}} \mathrm{F}_{\mathrm{i}}^{\mathrm{T}}$. If $\tilde{\mathrm{P}}^{-1}$ is expressed as $\tilde{\mathrm{P}}^{-1}=\left[\begin{array}{ll}\mathrm{P}_{11}^{*} & \mathrm{P}_{12}^{*} \\ \mathrm{P}_{21}^{*} & \mathrm{P}_{22}^{*}\end{array}\right]$, one obtains:

$\overline{\mathrm{R}}_{\mathrm{i}}=\overline{\mathrm{T}} \tilde{\mathrm{R}}_{\mathrm{i}}=\left[\begin{array}{cc}\tilde{\mathrm{P}}_{11} & \tilde{\mathrm{P}}_{12} \\ 0 & \mathrm{I}_{\mathrm{p}}\end{array}\right]\left[\begin{array}{cc}\mathrm{P}_{11}^{*} & \mathrm{P}_{12}^{*} \\ \mathrm{P}_{21}^{*} & \mathrm{P}_{22}^{*}\end{array}\right]\left[\begin{array}{c}0 \\ \mathrm{I}_{\mathrm{p}}\end{array}\right] \mathrm{F}_{\mathrm{i}}^{\mathrm{T}}=\left[\begin{array}{c}0 \\ \mathrm{P}_{22}^{*} \mathrm{~F}_{\mathrm{i}}^{\mathrm{T}}\end{array}\right]$

and so property 2 is proved

If there exists a Lyapunov matrix $\tilde{\mathrm{P}}$ which satisfies constraints (8), then the matrix $\overline{\mathrm{P}}=\left(\overline{\mathrm{T}}^{-1}\right)^{\mathrm{T}} \tilde{\mathrm{P}} \overline{\mathrm{T}}^{-1}$ is a Lyapunov matrix for $\overline{\mathrm{A}}_{0 \mathrm{i}}$ and satisfies the structural constraints $\quad \overline{\mathrm{C}}^{\mathrm{T}} \mathrm{F}_{\mathrm{i}}^{\mathrm{T}}=\overline{\mathrm{P}} \overline{\mathrm{R}}_{\mathrm{i}} \quad \forall \mathrm{i} \in\{1, \ldots, \mathrm{r}\}$. Using the partitioning of $\widetilde{\mathrm{P}}$ and $\overline{\mathrm{T}}$, a direct computation leads to:

$\overline{\mathrm{P}}=\left[\begin{array}{cc}\tilde{\mathrm{P}}_{11}^{-1} & 0 \\ -\tilde{\mathrm{P}}_{12}^{\mathrm{T}} \tilde{\mathrm{P}}_{11}^{-1} & \mathrm{I}_{\mathrm{p}}\end{array}\right]\left[\begin{array}{cc}\tilde{\mathrm{P}}_{11} & \tilde{\mathrm{P}}_{12} \\ \tilde{\mathrm{P}}_{12}^{\mathrm{T}} & \tilde{\mathrm{P}}_{22}\end{array}\right]\left[\begin{array}{cc}\tilde{\mathrm{P}}_{11}^{-1} & -\tilde{\mathrm{P}}_{11}^{-1} \tilde{\mathrm{P}}_{12} \\ 0 & \mathrm{I}_{\mathrm{p}}\end{array}\right]=\left[\begin{array}{cc}\tilde{\mathrm{P}}_{11}^{-1} & 0 \\ 0 & \overline{\mathrm{P}}_{2}\end{array}\right](12)$

where $\overline{\mathrm{P}}_{2}=\tilde{\mathrm{P}}_{22}-\tilde{\mathrm{P}}_{12}^{\mathrm{T}} \tilde{\mathrm{P}}_{11}^{-1} \tilde{\mathrm{P}}_{12}$ and thus $\overline{\mathrm{P}}$ has the required block diagonal structure of property 4 .

Finally, as the Lyapunov matrix $\overline{\mathrm{P}}$ related to $\overline{\mathrm{A}}_{0 \mathrm{i}}$ has been demonstrated to be block diagonal, the matrices $\overline{\mathrm{A}}_{011 \mathrm{i}}$ and $\overline{\mathrm{A}}_{022 \mathrm{i}}$ are stable. Indeed, from equations (2-a) and (12), one obtains:

$$
\begin{aligned}
& {\left[\begin{array}{ll}
\overline{\mathrm{A}}_{011 \mathrm{i}} & \overline{\mathrm{A}}_{012 \mathrm{i}} \\
\overline{\mathrm{A}}_{021 \mathrm{i}} & \overline{\mathrm{A}}_{022 \mathrm{i}}
\end{array}\right]^{\mathrm{T}}\left[\begin{array}{cc}
\overline{\mathrm{P}}_{1} & 0 \\
0 & \overline{\mathrm{P}}_{2}
\end{array}\right]+\left[\begin{array}{cc}
\overline{\mathrm{P}}_{1} & 0 \\
0 & \overline{\mathrm{P}}_{2}
\end{array}\right]\left[\begin{array}{cc}
\overline{\mathrm{A}}_{011 \mathrm{i}} & \overline{\mathrm{A}}_{012 \mathrm{i}} \\
\overline{\mathrm{A}}_{021 \mathrm{i}} & \overline{\mathrm{A}}_{022 \mathrm{i}}
\end{array}\right]<0} \\
& \Rightarrow\left\{\begin{array}{l}
\overline{\mathrm{A}}_{011 \mathrm{i}}^{\mathrm{T}} \overline{\mathrm{P}}_{1}+\overline{\mathrm{P}}_{1} \overline{\mathrm{A}}_{011 \mathrm{i}}<0 \\
\overline{\mathrm{A}}_{022 \mathrm{i}}^{\mathrm{T}} \overline{\mathrm{P}}_{2}+\overline{\mathrm{P}}_{2} \mathrm{~A}_{022 \mathrm{i}}<0
\end{array}\right.
\end{aligned}
$$

As $\quad \overline{\mathrm{A}}_{011 \mathrm{i}}=\overline{\mathrm{A}}_{11 \mathrm{i}}-\left(\overline{\mathrm{G}}_{\mathrm{i}} \overline{\mathrm{C}}\right)_{11}=\overline{\mathrm{A}}_{11 \mathrm{i}}$ and since $\left(\overline{\mathrm{G}_{\mathrm{i}}} \overline{\mathrm{C}}\right)_{11}=0$, $\forall \overline{\mathrm{G}}_{\mathrm{i}} \in \mathrm{R}^{\mathrm{n} \times \mathrm{p}}$ (see property 3 ), the matrices $\overline{\mathrm{A}}_{11 \mathrm{i}}$ are also stable, so property 1 is proved.

\subsection{Synthesis of a multiple observer}

Let us suppose that there exists a pair of Lyapunov matrices $\left(\widetilde{\mathrm{P}}, \tilde{\mathrm{Q}}_{\mathrm{i}}\right)$ checking the constraint (8) for each local model described by $\left(\tilde{\mathrm{A}}_{\mathrm{i}}, \tilde{\mathrm{B}}_{\mathrm{i}}, \tilde{\mathrm{R}}_{\mathrm{i}}, \widetilde{\mathrm{C}}\right)$. Then, there is a nonsingular transformation $\overline{\mathrm{T}}$ from which the multiple model with unknown inputs can be written in the following form:

$\left\{\begin{array}{l}\dot{\overline{\mathrm{x}}}(\mathrm{t})=\sum_{\mathrm{i}=1}^{\mathrm{r}} \mu_{\mathrm{i}}(\xi(\mathrm{t}))\left(\overline{\mathrm{A}}_{\mathrm{i}} \overline{\mathrm{x}}(\mathrm{t})+\overline{\mathrm{B}}_{\mathrm{i}} \mathrm{u}(\mathrm{t})+\overline{\mathrm{R}}_{\mathrm{i}} \overline{\mathrm{u}}(\mathrm{t})+\overline{\mathrm{D}}_{\mathrm{i}}\right) \\ \mathrm{y}(\mathrm{t})=\overline{\mathrm{x}}_{2}(\mathrm{t})\end{array}\right.$

or in a developed form:

$\dot{\overline{\mathrm{x}}}_{1}(\mathrm{t})=\sum_{\mathrm{i}=1}^{\mathrm{r}} \mu_{\mathrm{i}}(\xi(\mathrm{t}))\left(\overline{\mathrm{A}}_{11 \mathrm{i}} \overline{\mathrm{x}}_{1}(\mathrm{t})+\overline{\mathrm{A}}_{12 \mathrm{i}} \overline{\mathrm{x}}_{2}(\mathrm{t})+\overline{\mathrm{B}}_{1 \mathrm{i}} \mathrm{u}(\mathrm{t})+\overline{\mathrm{D}}_{1 \mathrm{i}}\right)$

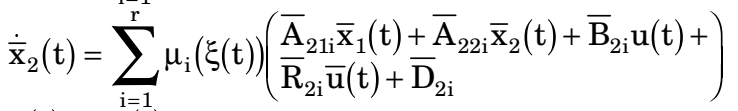

$\mathrm{y}(\mathrm{t})=\overline{\mathrm{x}}_{2}(\mathrm{t})$

Notice that $\bar{x}_{1}(t)$ does not depend explicitly upon the unknown inputs $\overline{\mathrm{u}}(\mathrm{t})$.

According to equation (13), the proposed multiple observer has the following form:

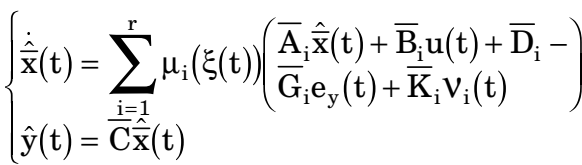

with $\overline{\mathrm{G}}_{\mathrm{i}}=\left[\begin{array}{c}\overline{\mathrm{A}}_{12 \mathrm{i}} \\ \overline{\mathrm{A}}_{22 \mathrm{i}}-\overline{\mathrm{A}}_{22}^{\mathrm{s}}\end{array}\right]$ and $\overline{\mathrm{K}}_{\mathrm{i}}=\left[\begin{array}{c}0 \\ \mathrm{P}_{2}^{-1} \overline{\mathrm{R}}_{2 \mathrm{i}}\end{array}\right]$

where $\hat{\overline{\mathrm{x}}}(\mathrm{t})$ represents the estimated state vector. $\overline{\mathrm{A}}_{22}^{\mathrm{s}}$ is a stable matrix and the discontinuous vector functions $v_{i}(t)$ are defined as follows:

$v_{i}(t)=\left\{\begin{array}{cc}-\rho E_{i}^{T}\left(E_{i} E_{i}^{T}\right)^{-1}\left\|e_{y}^{T}(t) P_{2} \bar{R}_{2 i}\right\| & \text { if } e_{y}(t) \neq 0 \\ 0 & \text { elsewhere }\end{array}\right.$

with: $\quad \mathrm{e}_{\mathrm{y}}(\mathrm{t})=\hat{\mathrm{y}}(\mathrm{t})-\mathrm{y}(\mathrm{t}), \mathrm{E}_{\mathrm{i}}=\mathrm{e}_{\mathrm{y}}^{\mathrm{T}}(\mathrm{t}) \overline{\mathrm{R}}_{2 \mathrm{i}}, \forall \mathrm{i} \in\{1, \ldots, \mathrm{r}\} \quad$ and $\mathrm{P}_{2} \in \mathrm{R}^{\mathrm{p} \times \mathrm{p}}$ is the unique symmetric positive definite solution of the Lyapunov equation:

$\mathrm{P}_{2} \overline{\mathrm{A}}_{22}^{\mathrm{s}}+\left(\overline{\mathrm{A}}_{22}^{\mathrm{s}}\right)^{\mathrm{T}} \mathrm{P}_{2}=-\mathrm{Q}_{2}$ 
Let us denote state estimation and output errors as $\mathrm{e}_{1}(\mathrm{t})=\hat{\overline{\mathrm{x}}}_{1}(\mathrm{t})-\overline{\mathrm{x}}_{1}(\mathrm{t})$ and $\mathrm{e}_{2}(\mathrm{t})=\mathrm{e}_{\mathrm{y}}(\mathrm{t})$. By direct time derivative, their dynamic evolutions check:

$$
\left\{\begin{array}{l}
\dot{\mathrm{e}}_{1}(\mathrm{t})=\sum_{\mathrm{i}=1}^{\mathrm{r}} \mu_{\mathrm{i}}(\xi(\mathrm{t})) \overline{\mathrm{A}}_{11 \mathrm{i}} \mathrm{e}_{1}(\mathrm{t}) \\
\dot{\mathrm{e}}_{2}(\mathrm{t})=\sum_{\mathrm{i}=1}^{\mathrm{r}} \mu_{\mathrm{i}}(\xi(\mathrm{t}))\left(\begin{array}{l}
\overline{\mathrm{A}}_{21 \mathrm{i}} \mathrm{e}_{1}(\mathrm{t})+\overline{\mathrm{A}}_{22}^{\mathrm{s}} \mathrm{e}_{2}(\mathrm{t})+ \\
\mathrm{P}_{2}^{-1} \overline{\mathrm{R}}_{2 \mathrm{i}} \mathrm{v}_{\mathrm{i}}-\overline{\mathrm{R}}_{2 \mathrm{i}} \overline{\mathrm{u}}(\mathrm{t})
\end{array}\right)
\end{array}\right.
$$

\section{Lyapunov equation}

In order to show the exponential convergence of this observer, let us consider the following Lyapunov function:

$$
\mathrm{V}\left(\mathrm{e}_{1}, \mathrm{e}_{2}\right)=\mathrm{e}_{1}^{\mathrm{T}} \mathrm{P}_{1} \mathrm{e}_{1}+\mathrm{e}_{2}^{\mathrm{T}} \mathrm{P}_{2} \mathrm{e}_{2}
$$

Its derivative in respect to time, evaluated along the trajectory of the system by using equations (2) and (16), may be expressed as:

$$
\dot{\mathrm{V}}=\sum_{\mathrm{i}=1}^{\mathrm{r}} \mu_{\mathrm{i}}(\xi)\left(\begin{array}{l}
\mathrm{e}_{1}^{\mathrm{T}}\left(\overline{\mathrm{A}}_{11 \mathrm{i}}^{\mathrm{T}} \mathrm{P}_{1}+\mathrm{P}_{1} \overline{\mathrm{A}}_{11 \mathrm{i}}\right) \mathrm{e}_{1}+ \\
\mathrm{e}_{2}^{\mathrm{T}}\left(\left(\overline{\mathrm{A}}_{22 \mathrm{i}}^{\mathrm{s}}\right)^{\mathrm{T}} \mathrm{P}_{2}+\mathrm{P}_{2} \overline{\mathrm{A}}_{22}^{\mathrm{s}}\right) \mathrm{e}_{2}+ \\
\mathrm{e}_{1}^{\mathrm{T}} \overline{\mathrm{A}}_{21 \mathrm{i}}^{\mathrm{T}} \mathrm{P}_{2} \mathrm{e}_{2}+\mathrm{e}_{2}^{\mathrm{T}} \mathrm{P}_{2} \overline{\mathrm{A}}_{21 \mathrm{i}} \mathrm{e}_{1}+ \\
2 \mathrm{e}_{2}^{\mathrm{T}} \mathrm{R}_{2 \mathrm{i}} \mathrm{v}_{\mathrm{i}}-2 \mathrm{e}_{2}^{\mathrm{T}} \mathrm{P}_{2} \overline{\mathrm{R}}_{2 \mathrm{i}} \overline{\mathrm{u}}
\end{array}\right)
$$

Proposition 2: there exists a symmetric positive definite matrix $\mathrm{P}_{2}$ checking (16), such that the dynamical errors (17) are asymptotically stable.

Proof: let $\mathrm{Q}_{1 \mathrm{i}} \in \mathrm{R}^{(\mathrm{n}-\mathrm{p}) \times(\mathrm{n}-\mathrm{p})}$ and $\mathrm{Q}_{2} \in \mathrm{R}^{\mathrm{p} \times \mathrm{p}}$ some definite positive matrices, and consider the matrices $\hat{\mathrm{Q}}_{\mathrm{i}} \in \mathrm{R}^{(\mathrm{n}-\mathrm{p}) \times(\mathrm{n}-\mathrm{p})}$ defined by:

$\hat{\mathrm{Q}}_{\mathrm{i}}=\overline{\mathrm{A}}_{21 \mathrm{i}}^{\mathrm{T}} \mathrm{P}_{2} \mathrm{Q}_{2}^{-1} \mathrm{P}_{2} \overline{\mathrm{A}}_{21 \mathrm{i}}+\mathrm{Q}_{1 \mathrm{i}}$

which are symmetric and definite positive too.

Let $P_{1} \in R^{(n-p) \times(n-p)}$ a symmetric positive definite matrix, unique solution of the Lyapunov equation (21).

$\overline{\mathrm{A}}_{11 \mathrm{i}}^{\mathrm{T}} \mathrm{P}_{1}+\mathrm{P}_{1} \overline{\mathrm{A}}_{11 \mathrm{i}}=-\hat{\mathrm{Q}}_{\mathrm{i}}$

The derivative (19) can be shown to be:

$$
\dot{\mathrm{V}}=\sum_{\mathrm{i}=1}^{\mathrm{r}} \mu_{\mathrm{i}}(\xi)\left(\begin{array}{l}
-\mathrm{e}_{1}^{\mathrm{T}} \hat{\mathrm{Q}}_{\mathrm{i}} \mathrm{e}_{1}-\mathrm{e}_{2}^{\mathrm{T}} \mathrm{Q}_{2} \mathrm{e}_{2}+\mathrm{e}_{1}^{\mathrm{T}} \overline{\mathrm{A}}_{21 \mathrm{i}}^{\mathrm{T}} \mathrm{P}_{2} \mathrm{e}_{2}+ \\
\mathrm{e}_{2}^{\mathrm{T}} \mathrm{P}_{2} \overline{\mathrm{A}}_{21 \mathrm{i}} \mathrm{e}_{1}+2 \mathrm{e}_{2}^{\mathrm{T}} \overline{\mathrm{R}}_{2 \mathrm{i}} \mathrm{v}_{\mathrm{i}}-2 \mathrm{e}_{2}^{\mathrm{T}} \mathrm{P}_{2} \overline{\mathrm{R}}_{2 \mathrm{i}} \overline{\mathrm{u}}
\end{array}\right)
$$

It is easy to verify that:

$$
\begin{aligned}
& \left(\mathrm{e}_{2}-\mathrm{Q}_{2}^{-1} \mathrm{P}_{2} \overline{\mathrm{A}}_{21 \mathrm{i}} \mathrm{e}_{1}\right)^{\mathrm{T}} \mathrm{Q}_{2}\left(\mathrm{e}_{2}-\mathrm{Q}_{2}^{-1} \mathrm{P}_{2} \overline{\mathrm{A}}_{21 \mathrm{i}} \mathrm{e}_{1}\right)= \\
& \left(\mathrm{e}_{2}^{\mathrm{T}} \mathrm{Q}_{2} \mathrm{e}_{2}-\mathrm{e}_{1}^{\mathrm{T}} \overline{\mathrm{A}}_{21 \mathrm{i}}^{\mathrm{T}} \mathrm{P}_{2} \mathrm{e}_{2}-\mathrm{e}_{2}^{\mathrm{T}} \mathrm{P}_{2} \overline{\mathrm{A}}_{21 \mathrm{i}} \mathrm{e}_{1}+\mathrm{e}_{1}^{\mathrm{T}} \overline{\mathrm{A}}_{21 \mathrm{i}}^{\mathrm{T}} \mathrm{P}_{2} \mathrm{Q}_{2}^{-1} \mathrm{P}_{2} \overline{\mathrm{A}}_{21 \mathrm{i}} \mathrm{e}_{1}\right)
\end{aligned}
$$

Taking into account (22), the expression of $\dot{\mathrm{V}}$ becomes:

$$
\dot{\mathrm{V}}=\sum_{\mathrm{i}=1}^{\mathrm{r}} \mu_{\mathrm{i}}(\xi)\left(\begin{array}{l}
-\mathrm{e}_{1}^{\mathrm{T}}\left(\hat{\mathrm{Q}}_{\mathrm{i}}-\overline{\mathrm{A}}_{21 \mathrm{i}}^{\mathrm{T}} \mathrm{P}_{2} \mathrm{Q}_{2}^{-1} \mathrm{P}_{2} \overline{\mathrm{A}}_{21 \mathrm{i}}\right) \mathrm{e}_{1}- \\
\tilde{\mathrm{e}}_{2 \mathrm{i}}^{\mathrm{T}} \mathrm{Q}_{2} \tilde{\mathrm{e}}_{2 \mathrm{i}}+2 \mathrm{e}_{2}^{\mathrm{T}} \overline{\mathrm{R}}_{2 \mathrm{i}} \mathrm{v}_{\mathrm{i}}-2 \mathrm{e}_{2}^{\mathrm{T}} \mathrm{P}_{2} \overline{\mathrm{R}}_{2 \mathrm{i}} \overline{\mathrm{u}}
\end{array}\right)
$$

with $\tilde{\mathrm{e}}_{2 \mathrm{i}}=\mathrm{e}_{2}-\mathrm{Q}_{2}^{-1} \mathrm{P}_{2} \overline{\mathrm{A}}_{21 \mathrm{i}} \mathrm{e}_{1}$.

By using the equation (20), the derivative of the Lyapunov function becomes:
$\dot{\mathrm{V}}=\sum_{\mathrm{i}=1}^{\mathrm{r}} \mu_{\mathrm{i}}(\xi)\left(-\mathrm{e}_{1}^{\mathrm{T}} \mathrm{Q}_{1 \mathrm{i}} \mathrm{e}_{1}-\tilde{\mathrm{e}}_{2 \mathrm{i}}^{\mathrm{T}} \mathrm{Q}_{2} \tilde{\mathrm{e}}_{2 \mathrm{i}}+2 \mathrm{e}_{2}^{\mathrm{T}} \overline{\mathrm{R}}_{2 \mathrm{i}} \nu_{\mathrm{i}}-2 \mathrm{e}_{2}^{\mathrm{T}} \mathrm{P}_{2} \overline{\mathrm{R}}_{2 \mathrm{i}} \overline{\mathrm{u}}\right)$

1. Let us suppose that the output error $e_{2}$ is different from zero. By using the expression (15) of $v_{i}$, the derivative of the function $\mathrm{V}$ becomes:

$\dot{\mathrm{V}}=\sum_{\mathrm{i}=1}^{\mathrm{r}} \mu_{\mathrm{i}}(\xi)\left(-\mathrm{e}_{1}^{\mathrm{T}} \mathrm{Q}_{1 \mathrm{i}} \mathrm{e}_{1}-\tilde{\mathrm{e}}_{2 \mathrm{i}}^{\mathrm{T}} \mathrm{Q}_{2} \tilde{\mathrm{e}}_{2 \mathrm{i}}-2 \rho\left\|\mid \mathrm{e}_{2}^{\mathrm{T}} \mathrm{P}_{2} \overline{\mathrm{R}}_{2 \mathrm{i}}\right\|-2 \mathrm{e}_{2}^{\mathrm{T}} \mathrm{P}_{2} \overline{\mathrm{R}}_{2 \mathrm{i}} \overline{\mathrm{u}}\right)$

As the unknown inputs are bounded, then:

$$
\begin{aligned}
\dot{\mathrm{V}} & \leq \sum_{\mathrm{i}=1}^{\mathrm{r}} \mu_{\mathrm{i}}(\xi)\left(-\mathrm{e}_{1}^{\mathrm{T}} \mathrm{Q}_{1 \mathrm{i}} \mathrm{e}_{1}-\tilde{\mathrm{e}}_{2 \mathrm{i}}^{\mathrm{T}} \mathrm{Q}_{2} \tilde{\mathrm{e}}_{2 \mathrm{i}}-2 \rho\left\|\mathrm{e}_{2}^{\mathrm{T}} \mathrm{P}_{2} \overline{\mathrm{R}}_{2 \mathrm{i}}\right\|+2 \rho\left\|\mathrm{e}_{2}^{\mathrm{T}} \mathrm{P}_{2} \overline{\mathrm{R}}_{2 \mathrm{i}}\right\|\right) \\
& =\sum_{\mathrm{i}=1}^{\mathrm{r}} \mu_{\mathrm{i}}(\xi(\mathrm{t}))\left(-\mathrm{e}_{1}^{\mathrm{T}} \mathrm{Q}_{1 \mathrm{i}} \mathrm{e}_{1}-\tilde{\mathrm{e}}_{2 \mathrm{i}}^{\mathrm{T}} \mathrm{Q}_{2} \tilde{\mathrm{e}}_{2 \mathrm{i}}\right)<0 \\
& \text { for }\left(\mathrm{e}_{1}, \tilde{\mathrm{e}}_{2 \mathrm{i}}\right) \neq 0, \forall \mathrm{i} \in\{1, \ldots, \mathrm{r}\}
\end{aligned}
$$

2. Let us suppose now that the output error $e_{2}$ is zero; the function $\dot{\mathrm{V}}$ is then written as:

$\dot{\mathrm{V}}=\sum_{\mathrm{i}=1}^{\mathrm{r}} \mu_{\mathrm{i}}(\xi)\left(-\mathrm{e}_{1}^{\mathrm{T}} \mathrm{Q}_{1 \mathrm{i}} \mathrm{e}_{1}-\tilde{\mathrm{e}}_{2 \mathrm{i}}^{\mathrm{T}} \mathrm{Q}_{2} \tilde{\mathrm{e}}_{2 \mathrm{i}}\right)<0$

for $\left(\mathrm{e}_{1}, \tilde{\mathrm{e}}_{2 \mathrm{i}}\right) \neq 0, \forall \mathrm{i} \in\{1, \ldots, \mathrm{r}\}$

Thus, we have demonstrated that the errors $\mathrm{e}_{1}(\mathrm{t})$ and $\mathrm{e}_{2}(\mathrm{t})$ tighten towards zero in an exponential way.

In conclusion, the multiple observer of the system (1) can be written as follows:

$\left\{\begin{array}{l}\dot{\hat{x}}(t)=\sum_{i=1}^{r} \mu_{i}(\xi(t))\left(\begin{array}{l}A_{i} \hat{x}(t)+B_{i} u(t)+D_{i}- \\ G_{i}(C \hat{x}(t)-y(t))+K_{i} v_{i}(t)\end{array}\right) \\ \hat{y}(t)=C \hat{x}(t)\end{array}\right.$

$\left\{\begin{array}{c}G_{i}=(\bar{T} \tilde{T})^{-1}\left[\begin{array}{c}\bar{A}_{12 i} \\ \bar{A}_{22 i}-\bar{A}_{22}^{s}\end{array}\right], \quad K_{i}=(\bar{T} \tilde{T})^{-1} P_{2}^{-1}(\bar{T} \tilde{T}) R_{i} \\ v_{i}(t)=\left\{\begin{array}{cc}-\rho E_{i}^{T}\left(E_{i} E_{i}^{T}\right)^{-1}\left\|e_{y}^{T}(t) P_{2} \bar{R}_{2 i}\right\| & \text { if } e_{y}(t) \neq 0 \\ 0 & \text { elsewhere }\end{array}\right.\end{array}\right.$

where

$\mathrm{e}_{\mathrm{y}}(\mathrm{t})=\mathrm{C} \hat{\mathrm{x}}(\mathrm{t})-\mathrm{y}(\mathrm{t})$ and $\mathrm{E}_{\mathrm{i}}=\mathrm{e}_{\mathrm{y}}^{\mathrm{T}}(\mathrm{t}) \overline{\mathrm{R}}_{2 \mathrm{i}}, \forall \mathrm{i} \in\{1, \ldots, \mathrm{r}\}$

\section{Example}

The selected nonlinear system is represented on figure 1 . It results from a traditional benchmark [6] and schematizes a hydraulic process made up of three tanks. These three tanks $\mathrm{T}_{1}, \mathrm{~T}_{2}$ and $\mathrm{T}_{3}$, with identical sections $\mathrm{A}$, are connected one to each other by cylindrical pipes with identical sections $S_{n}$. The output valve is located at the output of tank $\mathrm{T}_{2} ; \mathrm{T}_{2}$ it ensures to empty the tank filled by the pump flows 1 and 2 with respectively rates $Q_{1}(t)$ and $\mathrm{Q}_{2}(\mathrm{t})$. Two combinations of the three water levels are measured. The communication pipes between the tanks 
are equipped with manually adjustable ball valves, which allow the corresponding pump to be closed or open. The three levels $\mathrm{x}_{1}, \mathrm{x}_{2}$ and $\mathrm{x}_{3}$ are governed by the constraint $\mathrm{x}_{1}>\mathrm{x}_{3}>\mathrm{x}_{2}$; the process model is given by the equation (24).

Indeed, taking into account the fundamental laws of conservation of the fluid, one can describe the operating mode of each tank; one then obtains a nonlinear model expressed by the following state equations [6]:

$$
\begin{aligned}
& \int \mathrm{A} \frac{\mathrm{dx} \mathrm{x}_{1}(\mathrm{t})}{\mathrm{dt}}=\mathrm{Q}_{1}(\mathrm{t})-\alpha_{1} \mathrm{~S}_{\mathrm{n}}\left(2 \mathrm{~g}\left(\mathrm{x}_{1}(\mathrm{t})-\mathrm{x}_{3}(\mathrm{t})\right)\right)^{1 / 2} \\
& \mathrm{~A} \frac{\mathrm{dx}_{2}(\mathrm{t})}{\mathrm{dt}}=\mathrm{Q}_{2}(\mathrm{t})+\alpha_{3} \mathrm{~S}_{\mathrm{n}}\left(2 \mathrm{~g}\left(\mathrm{x}_{3}(\mathrm{t})-\mathrm{x}_{2}(\mathrm{t})\right)\right)^{1 / 2}- \\
& \left\{\alpha_{2} \mathrm{~S}_{\mathrm{n}}\left(2 \mathrm{~g}\left(\mathrm{x}_{2}(\mathrm{t})\right)\right)^{1 / 2}\right. \\
& \mathrm{A} \frac{\mathrm{dx}_{3}(\mathrm{t})}{\mathrm{dt}}=\alpha_{1} \mathrm{~S}_{\mathrm{n}}\left(2 \mathrm{~g}\left(\mathrm{x}_{1}(\mathrm{t})-\mathrm{x}_{3}(\mathrm{t})\right)\right)^{1 / 2}- \\
& \alpha_{3} S_{n}\left(2 g\left(x_{3}(t)-x_{2}(t)\right)\right)^{1 / 2}+Q f(t)
\end{aligned}
$$

where $\alpha_{1}, \alpha_{2}$ and $\alpha_{3}$ are constants. Qf(t) denotes an additional mass flow caused by a leak that constitutes the unknown input and $\mathrm{g}$ is the gravity constant.

The multiple model (1), with $\xi(\mathrm{t})=\mathrm{u}(\mathrm{t})$, which approximates the nonlinear system (24), is described by:

$\left\{\begin{array}{l}\dot{\mathrm{x}}(\mathrm{t})=\sum_{\mathrm{i}=1}^{4} \mu_{\mathrm{i}}(\xi(\mathrm{t}))\left(\mathrm{A}_{\mathrm{i}} \mathrm{x}(\mathrm{t})+\mathrm{B}_{\mathrm{i}} \mathrm{u}(\mathrm{t})+\mathrm{R}_{\mathrm{i}} \overline{\mathrm{u}}(\mathrm{t})+\mathrm{D}_{\mathrm{i}}\right) \\ \mathrm{y}(\mathrm{t})=\mathrm{Cx}(\mathrm{t})\end{array}\right.$

The matrices $A_{i}, B_{i}, C$, and $D_{i}$ are calculated by linearizing the initial system (24) around different points chosen in the operating range of the system. Four local models have been selected in an heuristic way. That number guarantees a good approximation of the state of the real system by the multiple model.

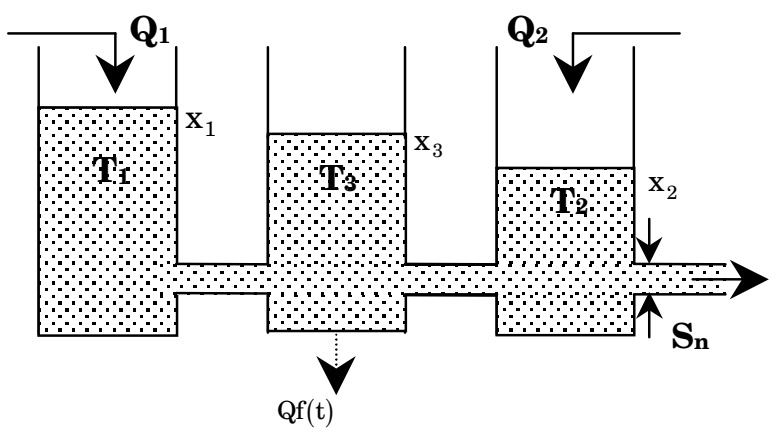

Schema 1 : Three tank system

\section{Simulation results}

The simulation results are represented on the following figures. The convergence of the state vector of the multiple observer towards those of the multiple model is quite good. At the vicinity of $t=0$, the disparity between estimated and actual state is due to the choice of initial conditions.
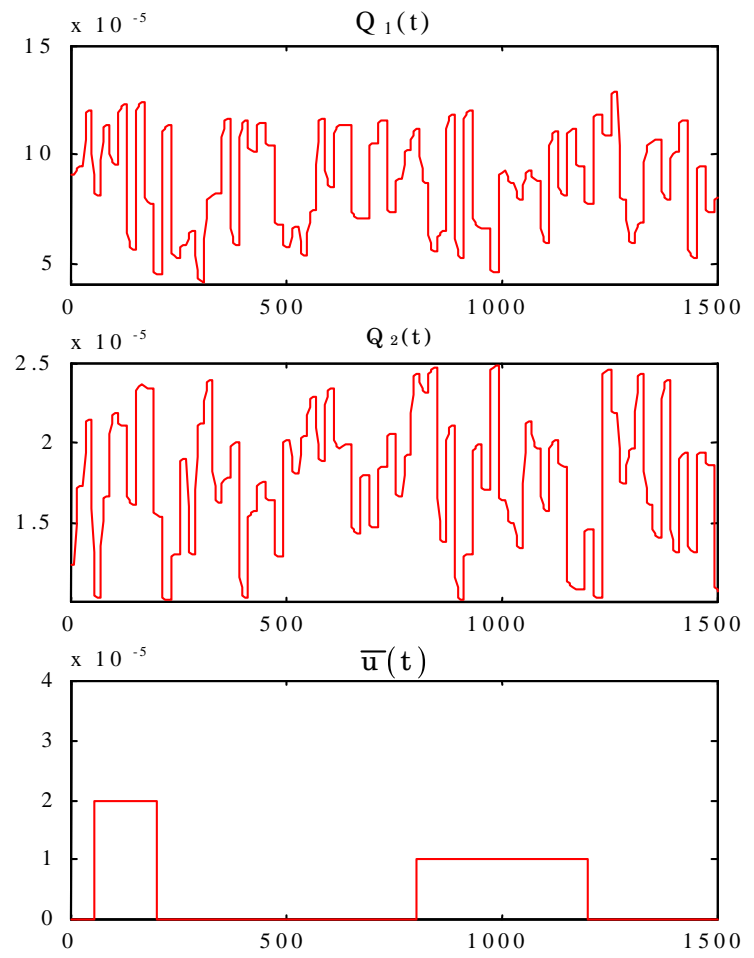

Figure 1: Multiple model inputs
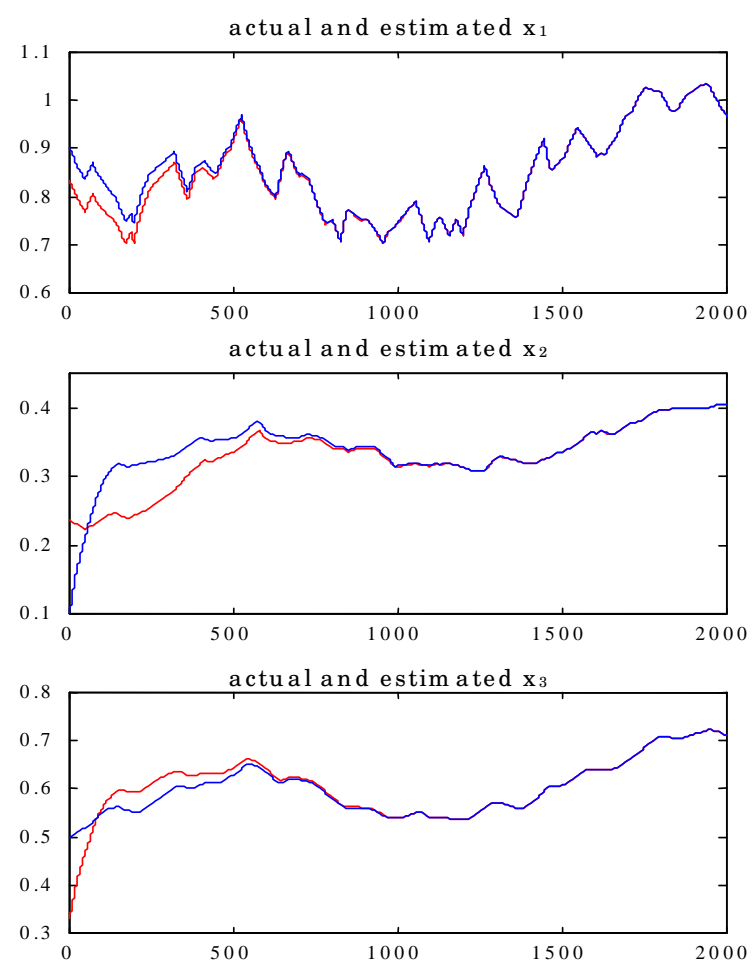

Figure 2: State estimation

\section{Conclusion}

In that paper, the design of a sliding mode non linear observer based on a multiple model has been proposed. The design of such observer relies on the existence of some matrices, namely $P, Q_{i}, F_{i}, i=\{1, \ldots, r\}$, ensuring, on one hand, the stability of the observer and, on second hand, satisfying a structural constraint allowing to isolate the unknown but bounded inputs in a particular 
part of the state vector.

Of course, the existence of such matrices depends on the number of unknown inputs with regards to the number of the measurements and the rank of the different associated matrices ; this point has not precisely been discussed in this paper because of space lacking. A first attempt of using this type of observer for fault detection and isolation has been presented on a well known three tank system. The quality of the obtained results seems to be sufficient to allow faults to be detected despite the presence of unknown inputs. Future works will deal with magnitude estimation of the unknown inputs.

\section{References}

1. C. Edwards and S. K. Spurgeon. On the development of discontinuous observers. Int. J. Control, 59 (5), pp. 1211-1229, 1994.

2. C. Edwards and S. K. Spurgeon. Sliding mode observers for fault detection and isolation. Automatica, 36 (4), pp. 541-553, 2000.

3. A. Akhenak, M. Chadli, D. Maquin, J. Ragot. Multiple observer with unknown inputs. Application to a three tank system. IAR Annual Meeting, ICD, 2002.

4. B. L. Walcott and S. H. Żak. Observation of dynamical systems in the presence of bounded nonlinearities/uncertainties. IEEE Conference on Decision and Control, CDC'1988, pp. 961-966 , 1988.

5. V. I. Utkin. Principles of identification using sliding regimes. Soviet Physic: Doklady, Sliding mode in control optimisation (Berlin: Springer-Verlag) 26, pp. 271-272, 1992.

6. A. Zolghadri, D. Henry, M. Monsion. Design of nonlinear observers for fault diagnosis: a case study. Control Engineering Practice, 4 (11), pp. 1535-1544, 1996.

7. R. J. Patton and J. Chen. Observer-based fault detection and isolation robustness and applications. Control Engineering Practice. 5 (5), pp. 671-682. 1997.

8. K. Gasso, G. Mourot and J. Ragot. Structure identification in multiple model representation: elimination and merging of local models. IEEE Conference on Decision and Control, CDC' 2001, Orlando, USA, December 2001.

9. T. A. Johansen, R. Shorten, R. Murray-Smith. On the interpretation and identification dynamic Takagi-Sugeno fuzzy models. IEEE Trans. on Fuzzy Systems, 8 (3), pp. 297-313, 2000.

10. Y. Blanco, F. Gouaisbaut, W. Perruquetti, P. Borne. Sliding mode controller design using polytopic formulation. IEEE Conference on Decision and Control, CDC' 2001, Orlando USA, December 2001.
11. Y. Guan, M. Saif. A novel approach to the design of unknown input observers. IEEE Trans. on Automatic Control, 36 (5), pp. 632-635, 1991.

12. D. Koenig, S. Mammar. Design of the class of reduced order unknown inputs nonlinear observer for fault diagnosis. American control conference, ACC'2001, Arlington, USA, June 2001. 